\title{
PLU-1/JARID1B overexpression predicts proliferation properties in head and neck squamous cell carcinoma
}

\author{
ZHIBIN CUI ${ }^{1,4^{*}}$, LIWEI SONG ${ }^{2 *}$, ZHAOYUAN HOU $^{5}$, YIFAN HAN $^{3}$, \\ YUHUA HU $^{6}$, YADI WU ${ }^{4}$, WANTAO CHEN ${ }^{1,4}$ and $\mathrm{LI} \mathrm{MAO}^{4,6}$ \\ Departments of ${ }^{1}$ Oral and Maxillofacial Surgery, ${ }^{2}$ General Surgery, and ${ }^{3}$ Oral Pathology, Ninth People's Hospital, \\ Shanghai Jiao Tong University School of Medicine, Shanghai 200011; ${ }^{4}$ Shanghai Key Laboratory of \\ Stomatology, Shanghai 200011; ${ }^{5}$ Department of Biochemistry and Molecular and Cellular Biology, \\ Shanghai Jiao Tong University School of Medicine, Shanghai, P.R. China; ${ }^{6}$ Department of Oncology and \\ Diagnostic Sciences, University of Maryland School of Dentistry, Baltimore, MD 20201, USA
}

Received November 24, 2014; Accepted February 19, 2015

DOI: 10.3892/or.2015.3849

\begin{abstract}
PLU-1/JARID1B (jumonji AT rich interactive domain $1 \mathrm{~B}$ ) is one of the testis cancer antigens and functions as a histone demethylase in the regulation of various human types of cancers. However, its functions in head and neck squamous cell carcinoma (HNSCC) are rarely reported. The aim of the present study was to examine PLU-1/JARID1B expression levels in HNSCCs and to investigate its role in cancer cell proliferation. In the present study, we found that PLU-1/JARID1B mRNA was upregulated in all tested HNSCC cell lines. Immunohistochemical staining showed that PLU-1/JARID1B protein expression was detected in $87.8 \%$ (87/99) of the HNSCC cases. A positive association was observed between high PLU-1/JARID1B expression and higher Ki-67 labeling in the HNSCC samples (Pearson $\mathrm{r}=0.6514, \mathrm{P}=0.0003)$. Stable PLU-1/JARID1B knockdown by PLU-1-shRNAs in the HNSCC cell lines suppressed cell growth both in the in vitro and in vivo studies. Moreover, PLU-1/JARID1B knockdown resulted in G1 arrest and early apoptosis by suppressing Bcl-2 family members in the HNSCCs. These data indicate that PLU-1/JARID1B is overexpressed in HNSCCs and is associated with tumor proliferation and apoptosis. Therefore, PLU-1/JARID1B represents a candidate proliferation biomarker for HNSCC diagnosis and treatment.
\end{abstract}

Correspondence to: Professor Li Mao, Department of Oncology and Diagnostic Sciences, University of Maryland School of Dentistry, 650 W. Baltimore Street, Baltimore, MD 21201, USA

E-mail:1mao@umaryland.edu

*Contributed equally

Key words: PLU-1/JARID1B, proliferation, apoptosis, head and neck squamous cell carcinoma, biomarker

\section{Introduction}

Head and neck squamous cell carcinoma (HNSCC) is one of the most common types of cancer worldwide (1). Despite advancements in the treatment of HNSCC over the past few decades, the clinical outcomes for advanced HNSCC patients remain dismal. Therefore, further elucidation of the molecular basis of HNSCC is critical for identifying molecular abnormalities that contribute to HNSCC progression. These abnormalities may serve as potential biomarkers for predicting clinical outcomes and/or molecular targets to improve the treatment of HNSCC patients.

Histone demethylases control the expression of downstream genes, which are known to promote cancer development $(2,3)$. PLU-1/JARID1B (jumonji AT-rich interactive domain $1 \mathrm{~B}$ ) is a member of the JARID1/KDM5 protein family. Similar to other members of the JARID1 family, PLU-1/JARID1B functions as a demethylase that is responsible for demethylation of lysine 4 of histone 3 trimethylation (H3K4me3) (4,5). PLU-1/JARID1B is highly expressed in testis tissues and in differentiated mammary glands, while expressed at low levels in other adult tissues (6-8). PLU-1/ JARID1B overexpression has been observed in breast cancer, which contributes to tumor cell proliferation (5,9-11). PLU-1/ JARID1B expression is also increased in bladder and lung cancers (12). The downregulation of PLU-1/JARID1B expression has been reported to repress cancer cell proliferation via regulating the E2F/RB1 cell cycle pathway (12). Roesch et al (13) reported that PLU-1/JARID1B could help characterize a subpopulation of slow-cycling cells in melanoma, revealing that PLU-1/JARID1B-positive cells are essential for continuous growth. Other groups have reported that KDM5 family members, including PLU-1/JARID1B, are highly expressed in embryonic stem cells $(14,15)$. These results suggest that PLU-1/JARID1B may play a role in maintaining stem cell properties in melanoma. As a testis/cancer-related gene with a potential role in stem cells, PLU-1/JARID1B may also contribute to the tumorigenesis and development of HNSCCs. 
The aim of the present study was to investigate the expression patterns and explore the functional role of PLU-1/JARID1B in HNSCCs.

\section{Materials and methods}

Patients and tissue. Ninety-nine HNSCC tissue samples were obtained from the Ninth People's Hospital of the Shanghai Jiao Tong University School of Medicine. Tissues were obtained from patients diagnosed with primary HNSCC from 1999 to 2009. Tumor tissues were stained with hematoxylin and eosin (H\&E) and classified histologically. Patients with HNSCC were staged according to the International Union Against Cancer TNM classification criteria (5th edition). Three normal oral mucosa tissues were obtained from the third molar extraction gingival tissue or palatal epithelial tissues during palatoplasty. The research protocol was reviewed and approved by the Institutional Review Board. All of the patients signed written informed consent in accordance with institutional guidelines.

Immunohistochemistry and evaluation. Tissue sections were deparaffinized in xylene, rehydrated in graded ethanol, treated with Tris-ethylene diamine tetraacetic acid buffer (for PLU-1/JARID1B) or citrate buffer (for Ki-67) for antigen retrieval and quenched in hydrogen peroxide. The tissue sections were then incubated overnight at $4^{\circ} \mathrm{C}$ with an antiPLU-1/JARID1B antibody (1:1,000 dilution; Abgent, San Diego, CA, USA) and $1 \mathrm{~h}$ at room temperature with a Ki-67 antibody (1:200 dilution; Shanghai Sunbio Co., Shanghai, China). The labeling index was defined as the intensity of staining $(0,1,2$ or 3$)$ multiplied by the percentage of positive tumor cells $(25,50,75$ or $100 \%)$. A pathologist examined 7 to 10 tumor areas in each section to calculate the final scores (ranging from 0 to 300). For PLU-1/JARID1B, scores marked as 0 were considered negative (negative staining), scores below 100 were marked as weakly positive (mild staining), and scores between 100 and 300 were marked as strongly positive (intense staining). For Ki-67, scores $>100$ were defined as Ki-67 high, and scores $\leq 100$ were considered Ki-67 low.

Cell culture. HNSCC cell lines (HN4, HN6, HN12, HN13 and HN30) were cultured in Dulbecco's modified Eagle's medium (DMEM; Gibco-BRL, Grand Island, NY, USA) supplemented with $10 \%$ heat-inactivated fetal bovine serum (FBS; Gibco-BRL), penicillin (100 U/ml), and streptomycin $(100 \mu \mathrm{g} /$ $\mathrm{ml})$ at $37^{\circ} \mathrm{C}$ in a humidified $5 \% \mathrm{CO}_{2}$ atmosphere. CAL27, SCC4, SCC9 and SCC25 cells (purchased from the American Type Culture Collection, Manassas, VA, USA) were cultured in DMEM/F12 medium (Gibco-BRL) supplemented with $10 \%$ heat-inactivated FBS, penicillin $(100 \mathrm{U} / \mathrm{ml})$ and streptomycin $(100 \mu \mathrm{g} / \mathrm{ml})$. Immortalized oral epithelial cells infected with human papillomavirus type 16-E6/E7 (HPV16E6/E7) were cultured in a defined keratinocyte serum-free medium (Gibco-BRL) (16). HIOEC cells, which are immortalized oral epithelial cells infected with human papillomavirus type 16-E6E7 (HPV16 E6/E7), were cultured in a defined keratinocyte serum-free medium (17). Normal human oral mucosa tissues were harvested from the area adjacent to the area of tooth extraction at the Oral and Maxillofacial Surgery Outpatient Clinic. The tissue was incubated with dispase overnight to separate the surface epithelium from the underlying fibrous connective tissue. Then, the epithelium was trypsinized to prepare a single-cell suspension. Primary normal human oral mucosa cells were cultured in the keratinocyte serum-free medium as previously described (18).

PCR and western blot analyses. Real-time PCR reactions were performed as previously described (19). The following primers were used to detect PLU-1/JARID1B and $\beta$-actin mRNA: 5'-TCAGTGCAGAGAGCCAGAGA-3' and 5'-ATCG AGGACACAGCACCTCT-3'; and 5'-CCTGGCACCCAGC ACAAT-3' and 5'-GGGCCGGACTCGTCATACT-3', respectively. The $\Delta \Delta \mathrm{Ct}$ method was applied to calculate the PLU-1/JARID1B expression levels in the HNSCC cell lines, as previously described (20). For western blotting, cells were lysed in radioimmunoprecipitation assay buffer (SigmaAldrich, St. Louis, MO, USA). The lysates were separated by sodium dodecyl sulfate-polyacrylamide gel electrophoresis. Primary antibodies against PLU-1/JARID1B (Proteintech, Chicago, IL, USA) were used to evaluate protein levels, and GAPDH or $\beta$-actin antibodies (Santa Cruz Biotechnology Inc., Santa Cruz, CA, USA) were used to normalize protein loading as previously described (20).

Generation of stable PLU-1/JARIDIB knockdown cell lines. Short-hairpin RNA (shRNA) synthetic oligodeoxynucleotides were cloned into the pLKO.1-puro vector (Sigma-Aldrich) according to the manufacturer's instructions. The sequences of the annealed oligonucleotides (against PLU-1/JARID1B) were as follows: sh14760-F, 5'-CCGGTCCTCTCCAAGATGTGG ATATActcgagTATATCCACATCTTGGAGAGGTTTTTG-3' and sh14760-R, 5'-AATTCAAAAACCTCTCCAAGATGT GGATATActcgagTATATCCACATCTTGGAGAGGA-3'; and 14761-F, 5'-CCGGTCCTGAGGAAGAGGAGTATCTTctcgag AAGATACTCCTCTTCCTCAGGTTTTTG-3' and 14761-R, 5'-AATTCAAAAACCTGAGGAAGAGGAGTATCTTctcgag AAGATACTCCTCTTCCTCAGGA-3'. Successful cloning and the orientation of the insert were validated by sequencing and PCR. PLU-1/JARID1B-shRNA-containing viral particles were produced in packaging cells (HEK 293T) after co-transfection with compatible packaging plasmids. Then, viral particle-infected HN4 and HN13 cells were selected with puromycin $(1.0 \mathrm{~g} / \mathrm{ml}$; Gibco) for 2 weeks to obtain stable PLU-1/JARID1B-knockdown cells.

Cell proliferation and colony formation assays. To analyze the proliferation potential of HN4 and HN13 cells stably expressing pLKO.1-puro-vector and pLKO.1-puro-PLU-1/JARID1BshRNA, cells were plated in 96 -well plates at $1 \times 10^{3}$ cells/ well and maintained at $37^{\circ} \mathrm{C}$ in a humidified incubator. At the indicated time-points (day 1, 3, 5, 7, 9 and 11), $10 \mu \mathrm{l}$ of CCK-8 solution (Dojindo Laboratories, Kumamoto, Japan) was added to the wells and incubated for $1 \mathrm{~h}$, and the absorbance was measured at $450 \mathrm{~nm}$ to calculate the number of viable cells in each well. Measurements were performed in quadruplicate, and the mean (standard deviation) optical density was reported.

Briefly, HN4 and HN13 (1x10 ${ }^{3}$ cells/plate) cells stably expressing PLU-1/JARID1B-shRNAs and pLKO.1-purovector were seeded in a $10-\mathrm{mm}$ plate and cultured in complete medium with $1 \mathrm{~g} / \mathrm{ml}$ puromycin for 2 weeks. Cell colonies were 
A

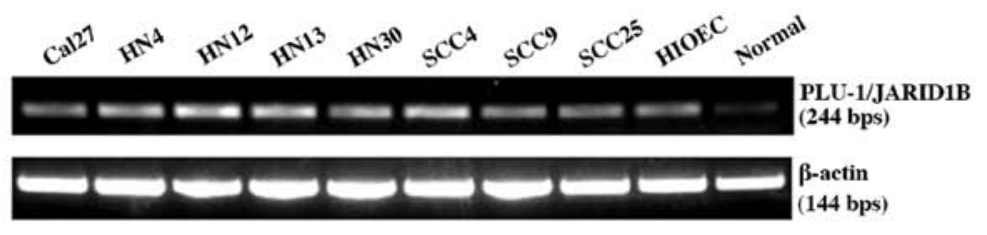

C

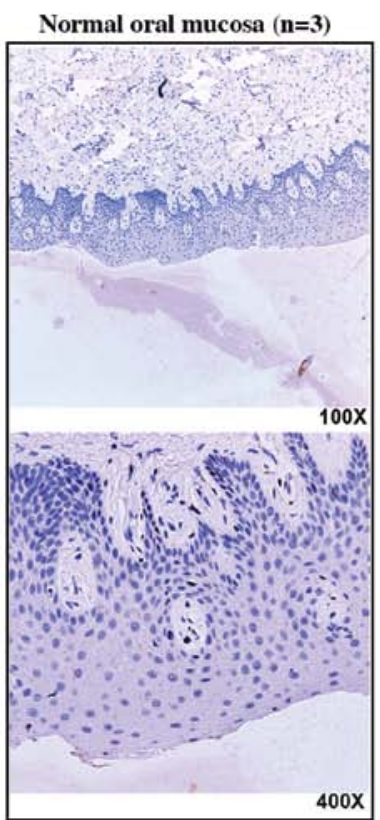

D Negative staining $(n=12$, scores $=0)$

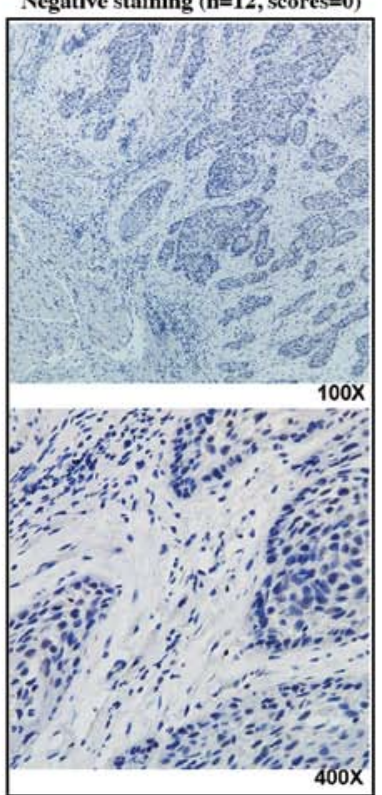

B

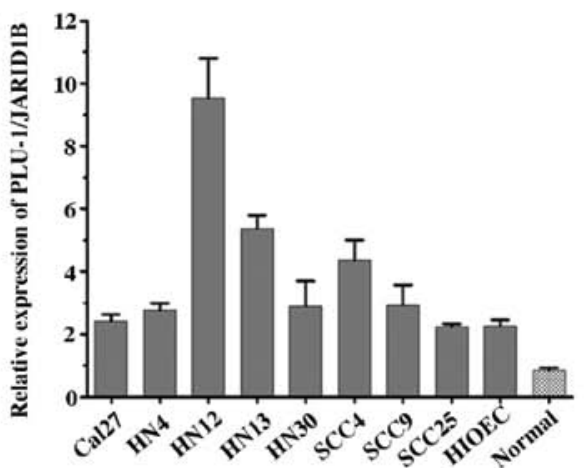

E Mild staining $(n=41$, scores $\leq 100)$

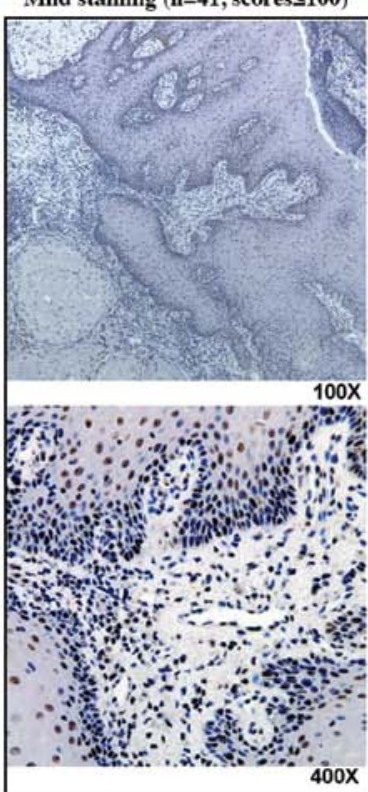

F Intense staining $(n=46$, scores $>100)$

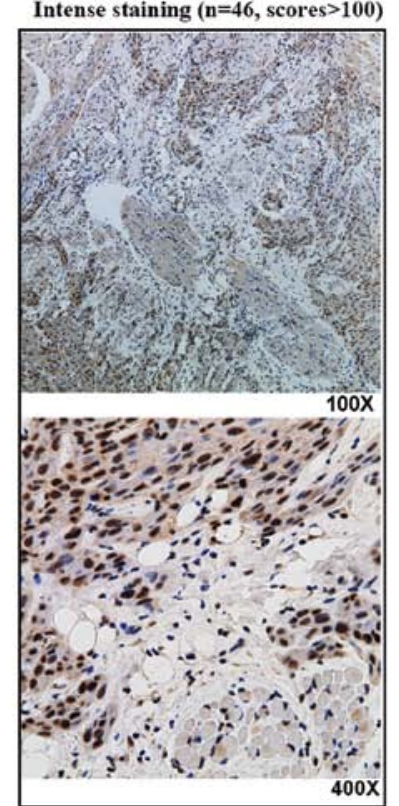

Figure 1. PLU-1/JARID1B is overexpressed in the HNSCC cell lines and patient samples. (A) RT-PCR for PLU-1/JARID1B mRNA expression in HNSCC cell lines. (B) Real-time-PCR for PLU-1/JARID1B mRNA expression in HNSCC cell lines. Results are means \pm SEM of three independent experiments normalized to normal mucosa sample. (C) Immunohistochemical staining for PLU-1/JARID1 in normal oral mucosa epithelium tissues. (D-F) Immunohistochemical staining for PLU-1/JARID1B expression in HNSCC patient samples. Different expression levels of PLU-1 are shown; upper and lower panels show x100 and x400 magnification, respectively.

visualized by staining with $0.25 \%$ crystal violet. After washing, the number of colonies containing $>50$ cells were counted.

Cell cycle and apoptosis assays. For cell cycle analysis, cells in the logarithmic growth phase were collected, and singlecell suspensions were permeabilized with $70 \%$ ethanol. Then, cells were labeled with propidium iodide (PI) and treated with RNase at $4^{\circ} \mathrm{C}$ for $30 \mathrm{~min}$. Apoptosis was assessed using Annexin V-PI staining without ethanol fixation, and the stained cells were quantified by flow cytometry using the Annexin V-FITC apoptosis detection kit (BD Biosciences, San Jose, CA, USA) according to the manufacturer's protocol (19).

In vivo tumor growth assay. Animal experiments were reviewed and approved by the Animal Ethics Committee of the Ninth Hospital, Shanghai Jiaotong University School of Medicine. Stable PLU-1/JARID1B-knockdown (shRNA no: sh14761) HN4 cells and stable control (PLKO.1-vector) HN4 cells were trypsinized and suspended in serum-free medium. Then, $1 \times 10^{7}$ cells from each group were bilaterally implanted subcutaneously into four nude mice (the right side received the control cells and the left side received the PLU-1-knockdown cells). Twenty days after inoculation, the mice were sacrificed, and the tumor volume was analyzed using the following formula: $\mathrm{V}=\left(\mathrm{A} \times \mathrm{B}^{2}\right) / 2$.

Statistical analysis. The data statistical analyses were performed using SPSS (standard version 17.0; SPSS Inc., Chicago, IL, USA). Real-time PCR analysis results were evaluated using the Mann-Whitney test for two independent groups. The results of the cell proliferation, colony formation assay, in vitro cell cycle, and apoptosis assays were evaluated using Student's t-tests. All tests were two-sided, and a P-value $<0.05$ was considered to indicate a statistically significant result.

\section{Results}

PLU-1/JARIDIB is overexpressed in the HNSCC cell lines. First, we examined the mRNA expression of PLU-1/JARID1B in 8 HNSCC cell lines, 1 immortalized oral epithelial cell 

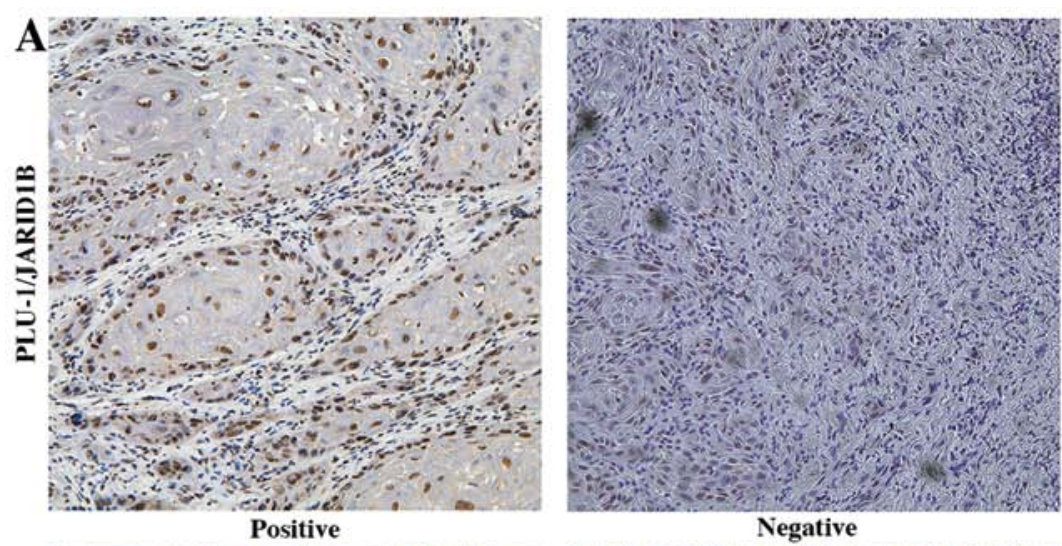

B
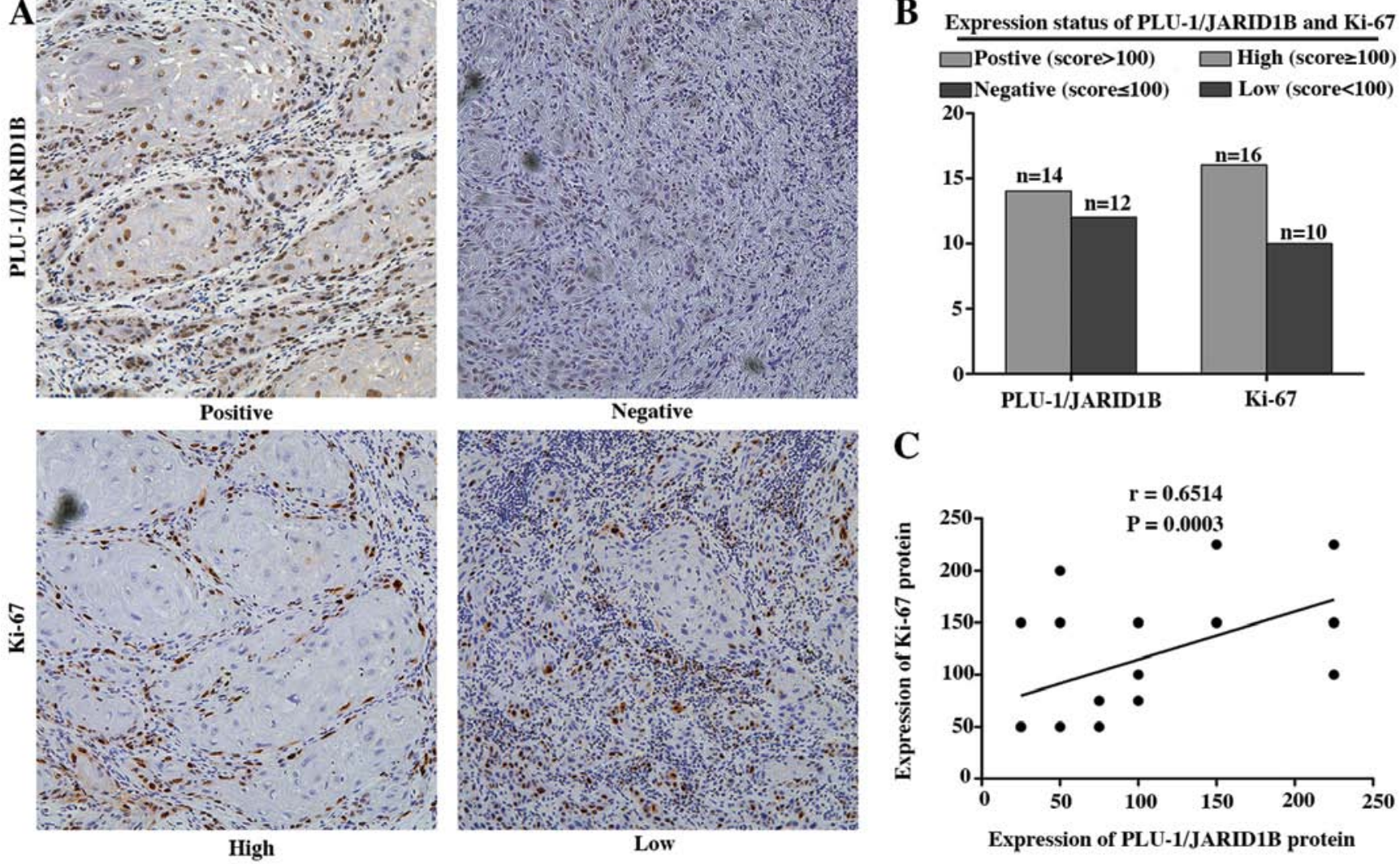

Figure 2. PLU-1/JARID1B overexpression is associated with high levels of Ki-67. (A) Immunohistochemical staining of PLU-1/JARID1B and Ki-67. (B) Case numbers of PLU-1 and Ki-67 expression levels. (C) Statistical analyses of the correlation between PLU-1 and Ki-67 expression.

line (HIOEC) and normal mucosa epithelium cells. The PLU-1/JARID1B transcription levels were higher in the HNSCCs and immortalized cell lines compared to the level in the primary cultured oral normal mucosa epithelium cells (Fig. 1A and B). We analyzed primary tumor tissues from 99 patients with HNSCC. PLU-1/JARID1B protein was expressed in $87(88 \%)$ of the tumors; 41 with low levels (scores $\leq 100$ ) and 46 with high levels (scores $>100$ ). In contrast, PLU-1/ JARID1B protein expression was not detected in all 3 normal oral mucosa epithelium tissues (Fig. 1C-F).

PLU-1/JARIDIB expression is associated with the Ki-67 labeling index. Notably, cells with high PLU-1/JARID1B expression were concentrated on the proliferating edge of the HNSCC tissues, leukoplakia and normal testis tissues (data not shown). To determine a potential association between PLU-1/JARID1B expression and HNSCC cell proliferation, we analyzed the expression pattern of PLU-1/JARID1B and the proliferation marker Ki-67 in a set of 26 HNSCC tissues. We observed a statistically significant positive association between PLU-1/JARID1B expression and Ki-67 indices [Pearson correlation coefficient $(\mathrm{r})=0.6514, \mathrm{P}=0.0003]$ (Fig. 2).

PLU-1/JARIDIB knockdown suppresses cell proliferation in vitro. To determine the potential involvement of PLU-1/JARID1B in HNSCC cell proliferation, we generated stable PLU-1/JARID1B-knockdown HN4 and HN13 cells using shRNAs. Among the five shRNAs tested, two markedly downregulated PLU-1/JARID1B expression levels in both cell lines (Fig. 3A and B). HN4 and HN13 cells with reduced PLU-1/JARID1B expression exhibited significantly lower growth compared to the control group (Fig. 3C and D). Consistent with this result, PLU-1/JARID1B downregulation significantly inhibited the colony formation in the HN4 $(\mathrm{P}<0.01)$ and HN13 $(\mathrm{P}<0.01)$ cells (Fig. 3E).

PLU-1/JARIDIB downregulation induces G1 arrest in HNSCC cells. To determine the mechanisms of how PLU-1/JARID1B downregulation inhibits HNSCC cell growth, we performed cell cycle analysis in the HN4 cells. FACS analysis revealed that PLU-1/JARID1B knockdown caused G1 arrest $(\mathrm{P}<0.05)$, with no changes in the $\mathrm{S}$ and $\mathrm{G} 2$ phases ( $\mathrm{P}>0.05$ ) (Fig. 4A). Moreover, the mRNA level of the G1 phase-related gene CCND2 was downregulated in the PLU-1/JARID1Bknockdown cells, whereas $\mathrm{P} 16^{\mathrm{INK} 4}$ was upregulated (Fig. 4B). These results suggest that PLU-1/JARID1B may facilitate G1 progression by regulating CCND2 and $\mathrm{P}^{1} 6^{\mathrm{INK} 4}$ expression.

PLU-1/JARIDIB is involved in the regulation of apoptosis. Annexin V and PI double labeling assays demonstrated that the number of early apoptotic cells was significantly higher in the PLU-1/JARID1B-knockdown cells compared to the controls $(\mathrm{P}<0.05)$ (Fig. 4C and D). Consistent with this result, western blot analyses indicated that Bak protein was upregulated, whereas the anti-apoptotic protein, Bcl-2, was downregulated in the PLU-1/JARID1B-knockdown cells (Fig. 4E). These data suggest that PLU-1/JARID1B maintains tumor cell survival by regulating Bcl family members. 
A
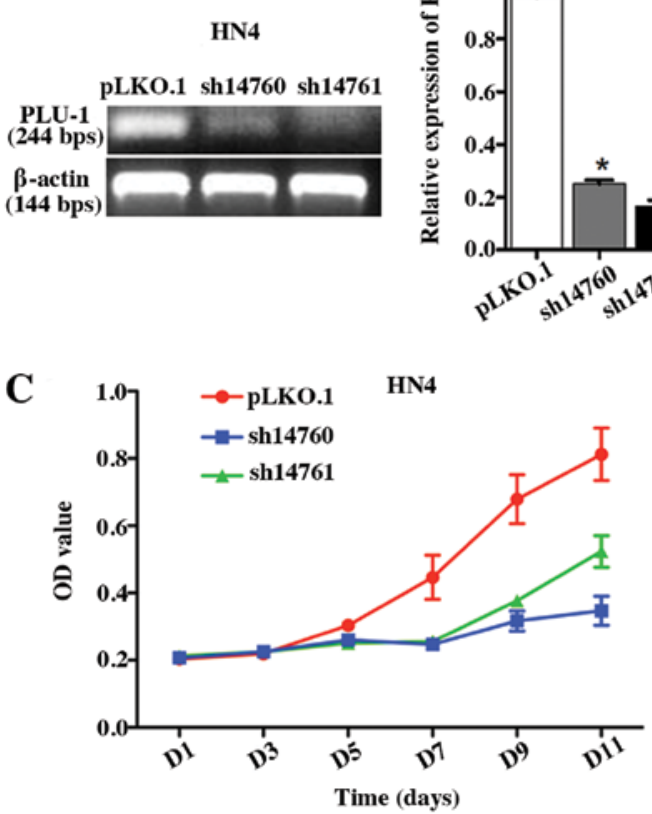

$\mathbf{E}$

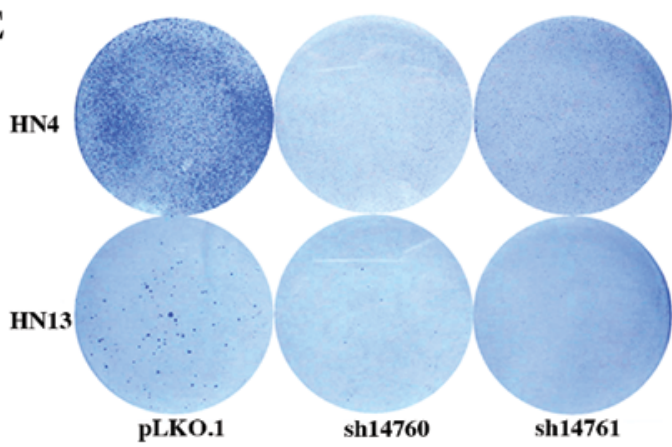

B

HN13

pLKO.1 sh14760 sh14761
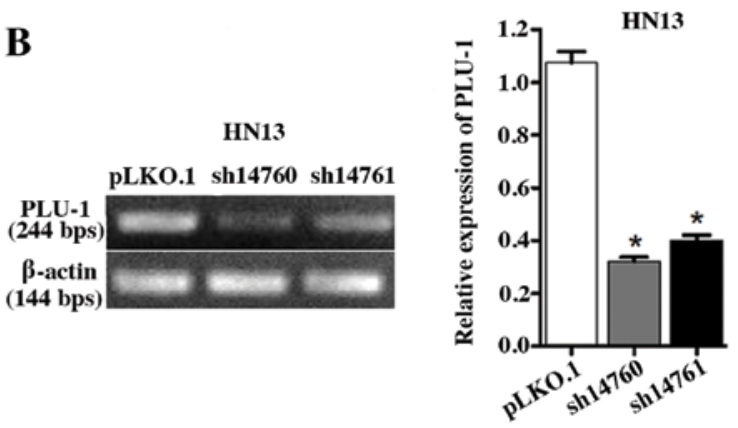

D
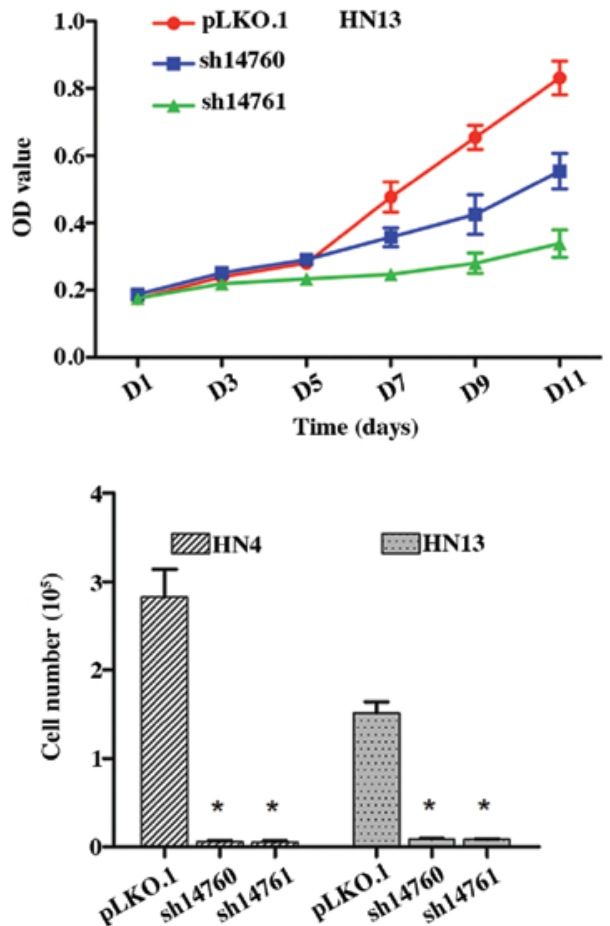

Figure 3. PLU-1/JARID1B knockdown suppresses cell proliferation in vitro. (A and B) Effects of PLU-1/JARID1B stable knockdown in HN4 and HN13 cells. PLU-1/JARID1B mRNA was examined using RT-PCR in the HNSCC cell lines. (C and D) Proliferation assays in stable PLU-1/JARID1B-knockdown and control HN4 and HN13 cells using Cell Counting kit-8 (CCK-8). (E) Colony formation assays in stable PLU-1/JARID1B-knockdown HN4 and HN13 cells. Results are the means \pm SEM of three independent experiments. " $\mathrm{P}<0.05$ vs. the control group.
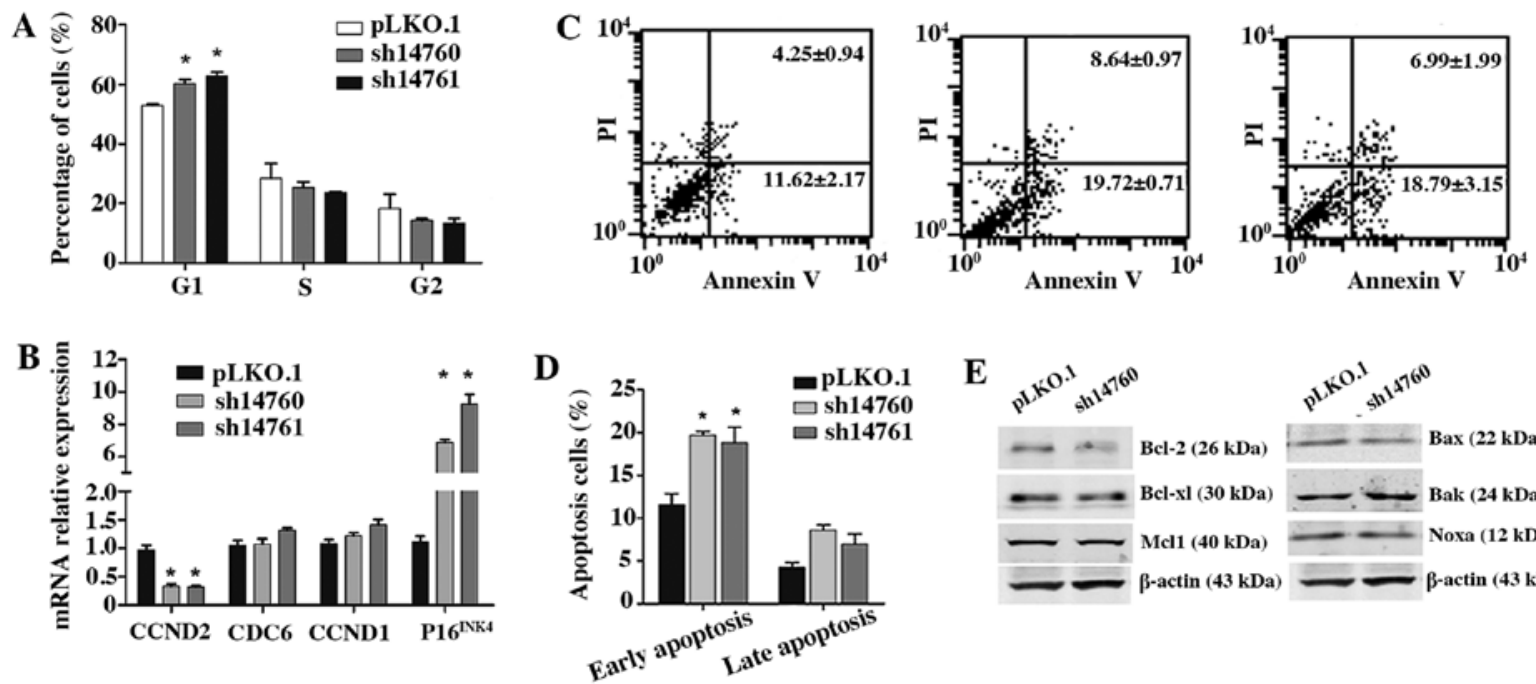

$\mathbf{E}$

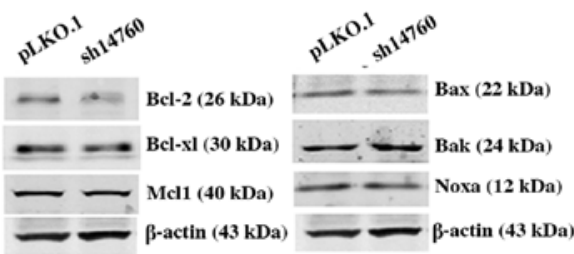

Figure 4. Cell cycle distribution and apoptosis were analyzed in the PLU-1/JARID1B stable knockdown cells. (A) Cell cycle analysis in stable PLU-1/JARID1Bknockdown HN4 cells via flow cytometry (FCM). (B) mRNA expression of G1-related genes was assessed using real-time-PCR. (C and D) Annexin V/PI staining was used to detect apoptosis in the PLU-1/JARID1B-knockdown HN4 cell line. (E) Western blotting for early apoptosis-related proteins. Results are the means \pm SEM of three independent experiments. "P $<0.05$ vs. the control group. 
A

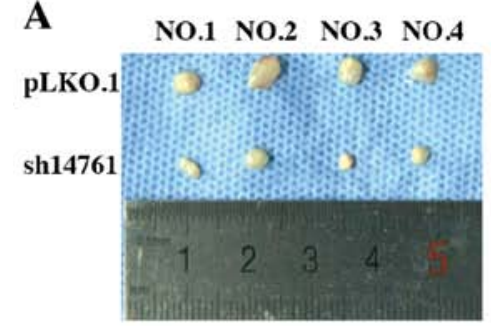

B

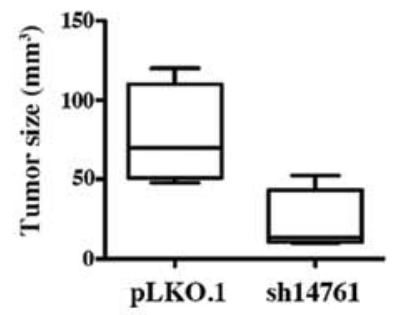

C
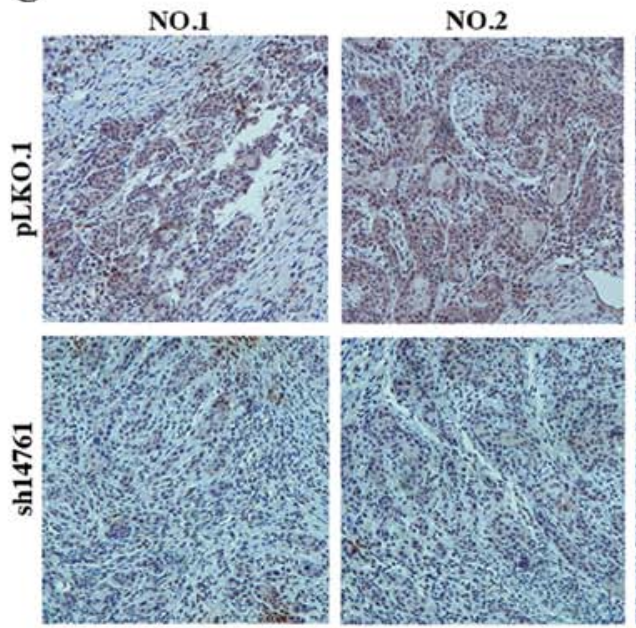
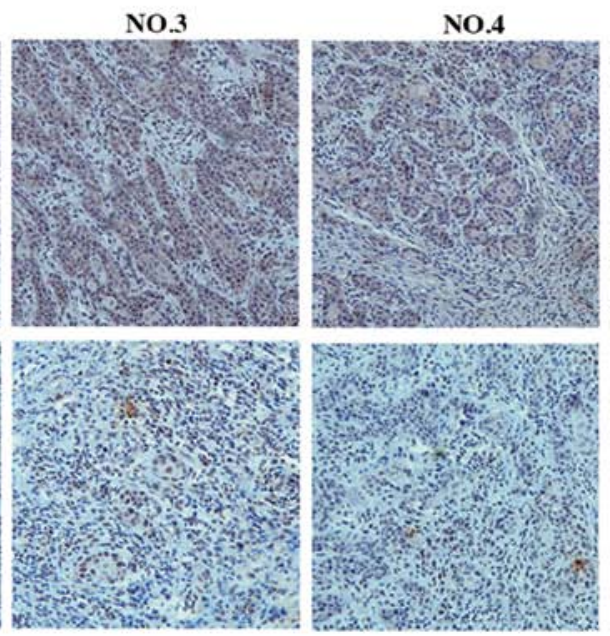

Figure 5. PLU-1/JARID1B knockdown suppresses cell growth in vivo. (A) In vivo tumor growth analysis in a nude mouse model. Stable PLU-1/JARID1Bknockdown HN4 cells were implanted subcutaneously in nude mice. (B) Tumor size analysis of the control and stable knockdown groups. Results are the means \pm SEM of triple measurements. ${ }^{*} \mathrm{P}<0.05$ vs. the control group. (C) Immunohistochemical staining of PLU-1/JARID1B protein in the xenograft tumors.

PLU-1/JARIDIB knockdown suppresses cell growth in vivo. Next, we used a nude mouse xenograft model to observe tumor formation in vivo. Six days after xenograft implantation, tumor nodes were observed in the control mice (HN4-PLKO.1-vector). Over 20 days, tumors in the PLU-1/JARID1B-knockdown group (HN4-sh14761) grew significantly slower in the nude mice compared to the control group. Thus, PLU-1/JARID1B silencing inhibited in vivo tumor growth (Fig. $5 \mathrm{~A}$ and $\mathrm{B}$ ). Immunohistochemical staining revealed that PLU-1/JARID1B protein was widely and strongly expressed in the tumors of the control group, whereas weak PLU-1/JARID1B expression was observed in the tumors of the PLU-1/JARID1B-knockdown group (Fig. 5C). These results suggest that PLU-1/JARID1B knockdown inhibited HNSCC growth in a subcutaneous xenograft nude mouse model.

\section{Discussion}

PLU-1/JARID1B is expressed in breast cancers but not in normal adult tissues at both the mRNA and protein levels (21). In the present study, we showed that PLU-1/JARID1B was highly expressed in all of the HNSCC cell lines tested as well as an immortalized oral epithelial cell line but not in primary cultured oral normal mucosa cells.

Lu et al (7) reported that PLU-1/JARID1B is only expressed in the adult testis and cancer tissues. We confirmed this finding in different cancer tissues and normal testis samples (data not shown), suggesting that PLU-1/JARID1B is a cancer/testis antigen. The aberrant expression of cancer/testis antigens is correlated with DNA promoter methylation status in different types of cancers $(22,23)$. In the present study, we observed that several transcription factors might bind to the PLU-1/JARID1B promoter and regulate its transcriptional activity, but further studies are needed to confirm this observation. Since the PLU-1/JARID1B promoter is GC rich, we hypothesized that promoter hypermethylation may block the binding of these transcription factors to DNA promoters in HNSCC, leading to the abnormal expression of PLU-1/JARID1B (24-26).
Similar to other cancer/testis antigens, such as MAGE, BAGE and GAGE, systemic PLU-1/JARID1B knockout led to early embryonic lethality in mice (9). Given the similarities between embryonic stem cells and cancer stem cells, it is possible that PLU-1/JARID1B may play a role in HNSCC progression and could serve as a diagnostic biomarker. Further studies are warranted to determine the stem cell-related properties of PLU-1/JARID1B.

The Ki-67 labeling index has been linked to cancer cell proliferation and poor clinical outcomes in HNSCC patients (27-29). We observed a positive association between PLU-1/JARID1B expression and Ki-67 labeling in HNSCC tissues. Unfortunately, the small sample size and incomplete follow-up data from the patient cohort prevented us from drawing a definitive conclusion for the potential associations between PLU-1/JARID1B expression and clinical parameters.

Roesch et al (13) reported that the expression pattern of PLU-1/JARID1B in HNSCC was quite different from the expression pattern in melanomas. They explained that this observation was because PLU-1/JARID1B is highly expressed in differentiated carcinoma cells, and epithelial carcinomas generally harbor more differentiated cells than melanomas. They concluded that PLU-1/JARID1B could be used as a biomarker for characterizing a subpopulation of slow-cycling melanoma cells. They also found that PLU-1/JARID1B knockdown initially accelerated tumor growth in melanoma cells, similar to the proliferative role of PLU-1/JARID1B protein observed in the present study. Thus, the potential cancer stem cell property of PLU-1/JARID1B warrants investigation, as it may represent another significant role for PLU-1/JARID1B in HNSCC progression. Researchers reported that PLU-1/JARID1B knockdown upregulated HOXA5 (5), which could induce p53 expression and promote apoptosis in breast cancer cells (30). In our PLU-1/JARID1B-knockdown HNSCC cells, Bcl-2 family members were regulated in response to decreased PLU-1/JARID1B expression.

In conclusion, PLU-1/JARID1B is overexpressed in HNSCCs and is associated with tumor proliferation. 
PLU-1/JARID1B knockdown induces tumor apoptosis by suppressing Bcl-2 family members. The mechanism underlying how these proteins are regulated by histone demethylases need to be studied in the future.

\section{Acknowledgements}

The present study was supported by the National Natural Science Foundation of China (30973343, 81072171 and 91229103) and by projects of the Shanghai Science and Technology Committee (11DZ2291800 and 10DZ1951300) and by Shanghai Jiaotong University School of Medicine Doctoral Innovation Foundation (BXJ201227).

\section{References}

1. Sun Q, Zhang J, Cao W, Wang X, Xu Q, Yan M, Wu X and Chen W: Dysregulated miR-363 affects head and neck cancer invasion and metastasis by targeting podoplanin. Int J Biochem Cell Biol 45: 513-520, 2013.

2. Amente S, Lania L and Majello B: The histone LSD1 demethylase in stemness and cancer transcription programs. Biochim Biophys Acta 1829: 981-986, 2013.

3. Jithesh PV, Risk JM, Schache AG, Dhanda J, Lane B, Liloglou T and Shaw RJ: The epigenetic landscape of oral squamous cell carcinoma. Br J Cancer 108: 370-379, 2013.

4. Huang PH, Chen $\mathrm{CH}$, Chou CC, Sargeant AM, Kulp SK, Teng CM, Byrd JC and Chen CS: Histone deacetylase inhibitors stimulate histone $\mathrm{H} 3$ lysine 4 methylation in part via transcriptional repression of histone H3 lysine 4 demethylases. Mol Pharmacol 79: 197-206, 2011.

5. Yamane K, Tateishi K, Klose RJ, Fang J, Fabrizio LA, ErdjumentBromage H, Taylor-Papadimitriou J, Tempst P and Zhang Y: PLU-1 is an H3K4 demethylase involved in transcriptional repression and breast cancer cell proliferation. Mol Cell 25: 801-812, 2007.

6. Barrett A, Santangelo S, Tan K, Catchpole S, Roberts K, SpencerDene B, Hall D, Scibetta A, Burchell J, Verdin E, et al: Breast cancer associated transcriptional repressor PLU-1/JARID1B interacts directly with histone deacetylases. Int J Cancer 121: 265-275, 2007.

7. Lu PJ, Sundquist K, Baeckstrom D, Poulsom R, Hanby A, MeierEwert S, Jones T, Mitchell M, Pitha-Rowe P, Freemont P and Taylor-Papadimitriou J: A novel gene (PLU-1) containing highly conserved putative DNA/chromatin binding motifs is specifically up-regulated in breast cancer. J Biol Chem 274: 15633-15645, 1999.

8. Madsen B, Spencer-Dene B, Poulsom R, Hall D, Lu PJ, Scott K, Shaw AT, Burchell JM, Freemont $P$ and Taylor-Papadimitriou J: Characterisation and developmental expression of mouse Plu-1, a homologue of a human nuclear protein (PLU-1) which is specifically up-regulated in breast cancer. Mech Dev 119 (Suppl 1): S239-S246, 2002.

9. Catchpole S, Spencer-Dene B, Hall D, Santangelo S, Rosewell I, Guenatri M, Beatson R, Scibetta AG, Burchell JM and TaylorPapadimitriou J: PLU-1/JARID1B/KDM5B is required for embryonic survival and contributes to cell proliferation in the mammary gland and in $\mathrm{ER}^{+}$breast cancer cells. Int $\mathbf{J}$ Oncol 38: 1267-1277, 2011

10. Coleman JA, Correa I, Cooper L, Bohnenkamp HR, Poulsom R, Burchell JM and Taylor-Papadimitriou J: T cells reactive with HLA-A*0201 peptides from the histone demethylase JARID1B are found in the circulation of breast cancer patients. Int J Cancer 128: 2114-2124, 2011.

11. Ohta K, Haraguchi N, Kano Y, Kagawa Y,Konno M, Nishikawa S, Hamabe A, Hasegawa S, Ogawa H, Fukusumi T, et al: Depletion of JARID1B induces cellular senescence in human colorectal cancer. Int J Oncol 42: 1212-1218, 2013.

12. Hayami S, Yoshimatsu M, Veerakumarasivam A, Unoki M, Iwai Y, Tsunoda T, Field HI, Kelly JD, Neal DE, Yamaue H, et al: Overexpression of the JmjC histone demethylase KDM5B in human carcinogenesis: involvement in the proliferation of cancer cells through the E2F/RB pathway. Mol Cancer 9: 59, 2010.
13. Roesch A, Fukunaga-Kalabis M, Schmidt EC, Zabierowski SE, Brafford PA, Vultur A, Basu D, Gimotty P, Vogt T and Herlyn M: A temporarily distinct subpopulation of slow-cycling melanoma cells is required for continuous tumor growth. Cell 141: 583-594, 2010.

14. Schmitz SU, Albert M, Malatesta M, Morey L, Johansen JV, Bak M, Tommerup N, Abarrategui I and Helin K: Jaridlb targets genes regulating development and is involved in neural differentiation. EMBO J 30: 4586-4600, 2011.

15. Stalker L and Wynder C: Evaluation of histone-modifying enzymes in stem cell populations. Methods Mol Biol 809: 411-426, 2012.

16. Zhong LP, Pan HY, Zhou XJ, Ye DX, Zhang L, Yang X, Chen WT and Zhang ZY: Characteristics of a cancerous cell line, HIOEC-B(a)P-96, induced by benzo(a)pyrene from human immortalized oral epithelial cell line. Arch Oral Biol 53: 443-452, 2008.

17. Cao W, Feng Z, Cui Z, Zhang C, Sun Z, Mao L and Chen W: Up-regulation of enhancer of zeste homolog 2 is associated positively with cyclin D1 overexpression and poor clinical outcome in head and neck squamous cell carcinoma. Cancer 118: 2858-2871, 2012.

18. Oda D and Watson E: Human oral epithelial cell culture. I. Improved conditions for reproducible culture in serum-free medium. In Vitro Cell Dev Biol 26: 589-595, 1990.

19. Cao W, Zhang ZY, Xu Q, Sun Q, Yan M, Zhang J, Zhang P, Han ZG and Chen WT: Epigenetic silencing of MAL, a putative tumor suppressor gene, can contribute to human epithelium cell carcinoma. Mol Cancer 9: 296, 2010.

20. Cui Z, Cao W, Li J, Song X, Mao L and Chen W: TRIM24 overexpression is common in locally advanced head and neck squamous cell carcinoma and correlates with aggressive malignant phenotypes. PLoS One 8: e63887, 2013.

21. Barrett A, Madsen B, Copier J, Lu PJ, Cooper L, Scibetta AG, Burchell $\mathrm{J}$ and Taylor-Papadimitriou J: PLU-1 nuclear protein, which is upregulated in breast cancer, shows restricted expression in normal human adult tissues: a new cancer/testis antigen? Int J Cancer 101: 581-588, 2002.

22. Glazer CA, Smith IM, Ochs MF, Begum S, Westra W, Chang SS, Sun W, Bhan S, Khan Z, Ahrendt S and Califano JA: Integrative discovery of epigenetically derepressed cancer testis antigens in NSCLC. PLoS One 4: e8189, 2009.

23. Yegnasubramanian S, Haffner MC, Zhang Y, Gurel B, Cornish TC, Wu Z, Irizarry RA, Morgan J, Hicks J, DeWeese TL, et al: DNA hypomethylation arises later in prostate cancer progression than $\mathrm{CpG}$ island hypermethylation and contributes to metastatic tumor heterogeneity. Cancer Res 68: 8954-8967, 2008.

24. Kim SG, Kim AS, Jeong JH, Choi JY and Kweon H: 4-Hexylresorcinol stimulates the differentiation of SCC-9 cells through the suppression of E2F2, E2F3 and Sp3 expression and the promotion of Sp1 expression. Oncol Rep 28: 677-681, 2012.

25. Kulkarni P, Shiraishi T, Rajagopalan K, Kim R, Mooney SM and Getzenberg RH: Cancer/testis antigens and urological malignancies. Nat Rev Urol 9: 386-396, 2012.

26. Zhao WF, Wang HB, Xie B, Hu LJ, Xu LH, Kuang BH, Li MZ and Zhang X: Sp1 and Sp3 are involved in the full transcriptional activity of centromere protein $\mathrm{H}$ in human nasopharyngeal carcinoma cells. FEBS J 279: 2714-2726, 2012.

27. Clahsen PC, van de Velde CJ, Duval C, Pallud C, Mandard AM, Delobelle-Deroide A, van den Broek L and van de Vijver MJ: The utility of mitotic index, oestrogen receptor and Ki-67 measurements in the creation of novel prognostic indices for node-negative breast cancer. Eur J Surg Oncol 25: 356-363, 1999.

28. Colozza M, Azambuja E, Cardoso F, Sotiriou C, Larsimont D and Piccart MJ: Proliferative markers as prognostic and predictive tools in early breast cancer: where are we now? Ann Oncol 16: 1723-1739, 2005

29. Trihia H, Murray S, Price K, Gelber RD, Golouh R, Goldhirsch A, Coates AS, Collins J, Castiglione-Gertsch M, Gusterson BA; International Breast Cancer Study Group: Ki-67 expression in breast carcinoma: its association with grading systems, clinical parameters, and other prognostic factors - a surrogate marker? Cancer 97: 1321-1331, 2003.

30. Raman V, Martensen SA, Reisman D, Evron E, Odenwald WF, Jaffee E, Marks J and Sukumar S: Compromised HOXA5 function can limit p53 expression in human breast tumours. Nature 405: 974-978, 2000 